\title{
CS Research Square \\ Quantum Kerr- (A) Ds Galilean Myers- Perr Driven Gravitational Transformations for the Anti-COVID-19 RoccuffirnaTM Drug Design
}

loannis Grigoriadis ( $\square$ jgrigoriadis@biogenea.gr )

\section{Research article}

Keywords: QRFQFT based Lorentzian Cryptographic signatures, Quantum Kerr- (A) Ds Galilean MyersPerr driven gravitational transformations, anti-COVID-19, micro-BlackHole-Inspired RoccuffirnaTM evolutionary drug design

Posted Date: February 22nd, 2022

DOI: https://doi.org/10.21203/rs.3.rs-1161686/v1

License: (c) (1) This work is licensed under a Creative Commons Attribution 4.0 International License. Read Full License 


\section{Abstract}

General methods to quantize reference frame transformations, to a "superposition of coordinate transformations" have been previously introduced on an array of recent observations developed through

gravitational amplification of primeval density fluctuations generated in the exceedingly early phase of cosmic evolution. In this paper, we strongly combine machine learning characteristics to Quantum Kerr(A) dS-Myers-Perry black microBlackHole-Inspired Gravitational for both Euclidean and Lorentzian signatures in Practice. I provide algorithms by means of mean percentile free energy ranking, in a new recall-based evaluation metric for the generation of an anti-COVID-19 small molecule combination of RoccuffirnaTM, RoccuttirnaTM, and EplerotiffirnaTM anti- (nCoV-19) ligands. In this paper, I show that the notion of entanglement and superposition are observer-dependent features in quantum circuit reference frames including Galilean trans formation, and near-horizon symmetries ranging from supergravity theories to Lorentzian cryptographic signatures to enhance the RoccuffirnaTM's gravity to trap the SARSCOV-2 viruses in practice.

\section{Introduction}

The emergence of a novel coronavirus infection causing new Severe acute respiratory syndrome coronavirus 2 (SARS-CoV-2), coronavirus ( $\mathrm{nCoV}-19$ ) has brought tremendous impact on worldwide health, $(1,2,3,4,5)$ whilst the chemogenomic interactions between the virus and the human is widely recognized to be critical foundation in responding the current outbreak the of the COVID2019 disease. Systems using DNA as a one-time code pad in a steganographic approach have been described (15). In work by Gehani et al. they proposed use of DNA codes assembled from short oligonucleotide sequences, into one-time pads. They further assume that the one-time pads can be kept as a pre-shared secret. The approach relies on encoding the plaintext through a DNA substitution a bit-wise XOR function between the plaintext and the DNA sequence. They also propose that the language for creating the DNA ciphertext be disjoint from the plaintext. Gehani also proposes an approach with biological instantiation (5). $(6,7,8,9,10,11$, 12) The virus has been named severe acute respiratory syndrome-coronavirus 2 (SARS-CoV-2) (3) because the RNA genome is about $82 \%$ identical to that of the SARS coronavirus (SARS-CoV) ; both viruses belong to clade $b$ of the genus Beta-coronavirus $(1,2)$ caused an outbreak of severe pulmonary disease in China, in the city of Wuhan, the capital of Hubei province and was initially detected in Himalayan palm civets (Guan et al., 2003) that may have served as an amplification host. The civet virus contained a 29-nucleotide sequence not found in most human isolates that were related to the global epidemic and thus, can be is considered as druggable targets. $(1-4,13,14,15,16)$ It has been speculated that the function of the affected open reading frame (ORF 10) might have played an important role in the trans-species jump infections. $(17,18,19,20,21)$ A similar virus was found later in horseshoe bat (13-20, $21,22,23,24$, and 25). Structural and biochemical characterizations have indicated to us that a 29-bp insertion in ORF 8 of bat-SARS-CoV genome, not found in most human SARS-CoV genomes of a common ancestor with civet SARS-CoV $(11-17,18-23,24,25,26,27)$. Numerical investigations and Equilibrium black-hole cryptography solutions to Einstein's Eqs have been known since the advent of general 
relativity. DNA signatures based on integration sites between the transgene insert and the flanking DNA make use of the same idea. While these types of signatures have been the paradigm of GMO detection for decades, this article strongly challenges the function of such signatures, especially relative to intended manipulations. Both traditionally and in the cyber-domain, signatures have long served as a valuable tool to guarantee the integrity and authenticity of the document being signed. However, the very concept of signatures in the cyber-realm first needed to be redressed as the Internet is Susceptible to intrusions that are not existent in the traditional setting. Analogously, it is argued here, that unique signature vulnerabilities exist in the biologic domain. A very recent study (Mueller, 2019) demonstrates that the existing DNA signature paradigm may be exploited via previously unrecognized for ms of attack $(5)$. $(29,30)$ By studying geometrical and mathematical principles of the quantum fields in a blackhole background, on the geometric framework Hawking demonstrated that this is not a mere analogy and in fact quantum mechanically black holes in which astrophysical processes occur, are a thermodynamic system. (29, 30, 31, 32) (Quantitative) Structure-Activity Relationships ((Q) SARs) Tools for artificial intelligence and data mining can derive in an objective and reproducible approximation of relative quantities and topological descriptors for finding eigenvectors, eigenvaluesby applying general solutions of the wave equationof Lamarckian-Laplacian paired with advanced machine learning algorithmsto for mulate an extension of the weak equivalence principle in chemical entities for generalizing small molecules into multi-targeted scaffolds. $(30,31,32,33)$ The state of a physical system has no absolute quantum meaning but is only defined relative to pharmacophoric reference framesin a special-relativistic or in a general-relativistic context when describing the motion of the ligand-receptor system. The same system may be associated to different states in different reference frames, which are normally related via some reference frame trans formation. From a physical point of view, a frame of reference is an abstraction of an idealized physical system: for example, an ideal rigid body can serve as a reference frame to define relative spatial distances and orientations of other objects. In classical physics, a coordinate transformationis used to trans for $\mathrm{m}$ the description of the system under consideration between two different reference frames. These transformations include, for example, spatial rotations and translations in space and time or constant relative motion of the frames (e. g., Galilean transformations). In general, the dynamical physical laws are invariant under some group of transformations. for instance, the laws of non-relativistic physics are invariant under Galilean transformations. In every physical laboratory situation, the reference frame is realized through a physical system. As any physical system, it ultimately behaves according to the laws of quantum mechanics. Therefore, one might see the standard treatment of reference-frame transformations as an approximation to a more fundamental set of transformations. Specifically, one should consider the possibility that one laboratory, from the perspective of another laboratory, might appear in a superposition or even become entangled with the system. Hence, the relationship between the two laboratories becomes more than a simple coordinate transformationbetween classical reference frames; it becomes a fundamentally quantum relationship. We may then speak about transformations between quantum reference frames (QRFs). for example, we can imagine that common virtual screening method and the fragmentizing of one chemical scaffold are fixed to a multi-targeted chemical entity that is in a superposition of position states with respect to the reference frame of other approved FDA small 
molecules. Can we meaningfully define transformations between such QRFs? Which transformations relate quantum states of pharmacophoric systems defined with respect to one chemical frame of reference to those pharmacophoric characters defined with respect to a second frame of reference? What are the black-hole solutions and dynamical physical laws are relatively invariant under such quantum symmetrical transformations? In this article, we discuss the various ways, singularities, and quantum in formation numerical methods about extremely specific molecular modeling problems to symmetrize chemical models where as extremal black hole geometries allowed us toaddress the composition, organization, and dynamics of a quantum reference frame in a nanoscale, for the computation of topological links and tangles and invariants of knots, through a stochastic discrete optimization procedure to rule out possible topologies, in diverse dimensions and theories. $(32,33,34,35,36,37)$ We also investigated lead optimization docking protocolsin quantum mechanics, that relies on the noiseless Subsystem reference frame method in combination with idealized 2D chemical symmetries to simplifying free energy assumptions with either idealized symmetries regarding the entropy-driven docking behavior, the new ligand's pharmacometrics behavior, and the protein-ligand interactions among the protein-ligand complexes (38). $(34,35,36,37,38,39,40)$ Our technique is motivated by a Bayesian based approach in quantum reference frames with in a quantum state as an outside for ce that tries to intercept and read the encoded chemical datawhich sometimes rendered biased fingerprintfragment where as Einstein's chaotic as well Mixmaster behaviors can be studied in the context of Hamiltonian dynamics, with the Hamiltonian $2 \mathrm{H}=-\mathrm{p} 2 \Omega+\mathrm{p} 2++\iiint \mathrm{Z} \mathrm{M} \iiint \mathrm{CS}(\mathrm{A}) \cos \theta j++-\iiint \mathrm{ZM} \iiint \mathrm{CS}(\mathrm{A}) \cos \theta j++\mathrm{p} 2-$ $+\mathrm{e} 4 \Omega(\mathrm{V}-1)$, to protect quantum states against undesired noise in terms of quantitative structure-activity relationship (QSAR). $(38,39,40,41,42,43)$ Here, for the first time we developed relational theories that predicts a fundamental decoherence mechanism by combining statistical significance, black-hole cryptography solutions to Einstein's Eqs and putative visualizations of time emerges from a timesymmetric theory on virtual compound libraries for per for ming quantum cryptographic communications in a quantum system relative to the quantum reference frame for pharmacophoric systems generally. (3142,43 ) Moreover, our model circumvents the problem of the - collapse of the wave packet\| as the probability interpretation is only ever applied to diagonal density operators. $(30,31-37)$ Turning to the dynamics, we propose an extension of the notion of covariance of the physical laws to include genuine quantum transformations, where one frame of reference is in a superposition of different relative positions, momenta, or velocities with respect to another frame of reference for generalizing chemical characters in a quantum pharmacophoric system. Here we investigated the conditions under which, in a quantum reference frame, and its own degrees of freedom, which can be in quantum superposition or entangled and evolve in time according to an account in terms of absolute quantities can provide a good approximation of relative quantities and topological descriptors for finding eigenvectors and eigenvalues of the combinatorial Lamarckian-Laplacian paired with advanced machine learning algorithms, such as the data mining, Al-Quantum computing, entanglement complexity guidelines for (Q) SAR requirements as well as per for mance implications, such as deep neural network (DNN), random for est (RF), and gradient boosting decision tree (GBDT), to facilitate their applications to quantitative toxicity and fragment based drug design predictions. $(34,35,36-37)$ In this hybrid drug designing approach, we applied an operational formalism black-hole solutions to Einstein's Eqs for per for ming relational 
reference frame theory quantum communications into neural matrix factorizations, cryptography, Schrödinger inspired docking algorithms, and other Lorentzian signatures driven Information-theoretic tasks to merge the pharmacophoric elements from different chemical and physical databases into the RoccuffirnaTM innovative structure to for $\mathrm{m}$ a rigid chemical scaffold with anti-COVID19 properties $(1,4-22,23-43,44)$.

\section{Methods And Materials}

Detailed methods are provided in the online version of this paper and include the following:

\section{RESOURCE AVAILABILITY}

Lead contact

Data and code availability

\section{EXPERIMENTAL MODEL AND SUBJECT DETAILS}

- Public Datasets, SARS-COV-2 motif peptide consensus strategy.

- Screening library and COVID2019 targets.

\section{METHOD DETAILS}

- Pharmacophoric-ODEs fragmentating,merging and recoring of the selected Hit compounds: Biogenetoligandorol Al-microBlackHole heuristic algorithm.

- Roccuffirna free pharmacophoric system

- Geometric symmetries from a quantum reference multi-merged pharmacophoric frame.

\section{Results}

In this computational drug design project we provided an extensive combination of toolboxes by applying black-hole solution to Einstein's Eqs for perfor ming quantum communication, neural matrix factorizations, cryptography, Schrödinger inspired docking algorithms, and other Information-theoretic tasks in MathCast programming language, and compared these algorithms by means of mean percentile free energy ranking, in a new recall-based evaluation metric for the Insilco design of a Novel Series of RoccuffirnaTMQMMMCoRoNNARRFr anti- (nCoV-19) annotated ligands. (Figure S1a). 3D Docking interactions of the selected NuBEE physical elements inside the PDB:6XS6, SARS-CoV-2 Spike D614G variant were combined to various general docking results including heuristic horizon topologies, into nearhorizon pharmacophoric fragmentations as applied to Euclidean symmetries ranging from applied supergravity theories to a system of intrinsically positioned cables. $(35,36,37,38,39)$ (Table S1). Docking energy rankings of the physical hit compoundsof Wyerone, bis- (5-for mylfurfuryl) ether, Monocrotaline, and Zeatin (Figure S1f), Methoxsalen, behenic acid, Bergapten, L-gamma -Glutamyl-S- 
allylthio-L-cysteine, Oleic Acid, Sursane, Hesperetin, Adenosine, and Eriodictyol (Figure S1g), Baicalein-7methyl ether, Euglobal III, and the Linoleic Acid residues (Figure S1h), Atrazine, Genistein, Pregnenolone ligands (Figure S1i) when docked onto the SARS-COV-2 protein targets were applied and kinematically sTabstriangular bars generated when filtered befor e evaluation as extracted from the selected physical chemical databases. Structurally valid symmetricformations were then connected into smaller molecule components, holes, $(40,41,42,43,44)$ and voidsjointed at their endsby hinged connections into Roccuffirna'schemical structure to trap computationally the SARS-COV-2 viruses in practice.

\section{Experimental docking results.}

(Figure S1b) Energy vibrations, micro-Black Hole Spin densities, and Electrostatic Potentials of the Roccuffirna small molecule were then generated using the MASK software, (Figure S2a) indicating that contact residues of our new QMMM designed small molecule are involved in the formation of water bridges, hydrogen bond, interactions, and metal complexes when reacted with the 03126602 ligand donor atoms and docked with the sequence of V-M-ASN-142, V-S-ASN-142, V-M-MET -165, V-S-MET-165, V -M-GLU-166, V-S-GLU-166, V-M-LEU-167, V-M-PRO-168 residues with the docking energy values of (-2. 95, $-3.73,-138.78,-4745,-33.183,-27.630,-6.449,-31.059) \mathrm{Kcalmol}$ inside the active site pocket of the (PDB:6xs6) protein targets respectively. (Figure S2b). Merged pharmacophoric elements of ((2S, 5R, 6R) -6- ((2S) -2-amino-2-phenylacetamido) - 3, 3-dimethyl-7- oxo- 4-thia-1- azabicyclo (3. 2. 0 ) heptane2- carbonyloxy), (\{ ((2-amino-6-oxo-6, 9-dihydro-3H- purin-9-yl) oxy), (hydroxy) phosphoryl\}oxy) phosphinic acid $\{((1 S, 4 S)$-5 -oxabicyclo (2. 1. 0) pentan-2ylidene) \{ (cyano (\{2, 6-diazabicyclo (3. 1. 0) hex- 1-en-6-yl\}) phosphanyl), (fluoro) methyl\} -lambda6sulfanyl\} one $\{((2 R, 3 S, 5 R)-3-((\{((2 R, 3 S, 5 R)-5-\{2$-amino-6- $((E)-\{((R)-($ aminooxy) $)((1 ' S, 1 " S, 3 S$, 3'S, 4S, 5"S) -3' - (aminooxy) -5"-\{N- ((1R) -1, 2- ((2S, 5R, 6R) -6- ((2S) -2-amino-2- phenylacetamido) -3, 3dimethyl-7-oxo- 4-thia-1-azabicyclo (3. 2. 0) heptane-2- carbonyloxy), (\{ ((2-amino-6-oxo-6, 9-dihydro-3Hpurin-9-yl) oxy) (hydroxy) phosphoryl\} oxy) phosphinic acid) formamido\}- residues of the RoccuffirnaTM small molecule generated a docking effect which is involved in the generation of hydrogen bondswhen reacted with the 474503126602 ligand donor atoms inside the DOZ A binding cavities of the amino acid sequence of V-S-GLU-166, V -M-LEU-167, V-M-PRO-168, V-S-PRO-168, V-M-ARG-188, V-M-GLN -189, VS-GLN-189 with the docking energies of $(-2.95,-3.73,-138.78,-33.183,-27.630,-6.449,-31$. 059) Kcalmol when docked onto the SARS-COV-2 active pockets of the (PDB:2g9t) protein targets while binding to Roccuffirna small molecule surfaces of the amino acid sequences of V-S-VAL-116, V-M-THR118, V-S-THR-118, V-M-PRO-84, V-S -PRO-84, V-M-ASN-85, V-M-ASN-114, V-M-THR-115, V-S-THR-115, V-MVAL-116 with a high docking energy score of $(-86.6$, and $-4.78161,-5.02437,-5.37485,-6.01569,-4$. $08585,-9.1855,-4.79454,-5.5449,-4.52233)$ Kcalmol respectively. (Figure S2C). The ((2S, 5R, 6R) -6((2S ) -2-amino-2-phenylacetamido) -3, 3-dimethyl-7-oxo- 4-thia-1-azabicyclo (3. 2. 0) heptane-2carbonyloxy) contact residues of the Roccuffirna chemical structure when docked onto the SARS-COV-2 protein electrostatic surface binding sites of the (PDB:3fqq) protein targets inside the binding cavities of the amino acid sequences of V-M-ARG-112, V-S-ARG-112, V -S-HIS -159, V-M-TYR-161, V -M-ARG-112, V-SHIS-159 generated in total a negative docking energy score of $-87.2 \mathrm{Kcalmol}$ with docking energy values of (-87. 2 and $-5.9744,-11.9828,-13.3388,-4.9979,-5.08108,-13.4218)$ KcalmolA respectively 
indicating that our QMMM designed prototype may be of a potent anti-viral inhibitory agent for the down regulation the expression levels of the novel dimeric form of NS5A (amino acids33 to 202) of the (NS5A (33 -202) ) domain I protein from hepatitis C virus. (Figure S2d) More 3D Docking visualizations between electrostatic regions and the Roccuffirna chemical structure have shown to us that ((2S, 5R, 6R) -6- ((2S) 2-amino- 2-phenylacetamid o) -3, 3-dimethyl-7-oxo- 4-thia-1-azabicyclo (3. 2. 0) heptane-2- carbonyloxy), (\{ ((2-amin o-6-oxo-6, 9-dihydro-3H-purin-9-yl) oxy) (hydroxy) phosphoryl\} oxy) phosphinic acid1' (4"), 1"-dien- 4-yl) phosphanyl) methylidene\}amino) - 3lambda4, 7lambda4, 9-lambda4-purin-9-yl\} -3$((\{((2 R, 3 S, 5 R)$ contact residues were able of producing hydrophobic interactions, and water bridges when docked inside the (PDB:7bv2) SARS-COV-2 protein targets with in the X77 A binding cavities of V-MASN-142, V-S-ASN-142, V-M-MET-165, V-S-MET-165, V-M-GLU-166, V -S-GLU-166, V-M-LEU-167, V-M-PRO168 amino acid regions with the docking energy values of $(-3.75,-4670,-609,-20.444,-13.613,-29.034$, $-19.778,-13.574,-32.721$ ) Kcalmol indicating that our drug novelty is able of generating 7,3 fold times of higher negative docking energies against the nsp12-nsp7-nsp8 complexes when compared to triphosphate for $m$ of the Remdesivir (RTP) active sites inside the same SARS-Cov-2 protein targets. (Table s2), (Table s3). (Figure S3a). Docking Energy rankings between Roccuffirna chemical structure and the other FDA approved drugs performed and a EWEIGHT-GENEX 3D Docking energy cluster analysis which was reproduced between Roccuffirna chemical structure with Remdesivir- (PDB:6lu7) ligand-protein complexes as co-factor indicated that our chemical design is able of generating total docking energies of 26.704 fold times of higher docking energy values when compared to Remdesivir and LopinavirRitonavir small molecules at the same (PDB:6lu7) protein target. The RoccuffirnaTM (Figure S3a), (Table s6) drug design was observed also of generating a multi-targeting inhibitory docking effect and generated negative docking energies into the binding sites of the protein targets of the (PDB:6yb7) with the docking energy values of (T. Energy, I. Energy, vdW, Coul, NumRotors, RMSD, Score), (-116. 717, -36. 220, -13. 116, -23. 104, 12, 7, 077, -7. 447) Kcalmol. The Remdesivir small molecule generated an agonistic binding effect and generated positive docking energies inside the binding sites of the protein targets of (PDB:1xak) with the docking energy values of (T. Energy, I. Energy, vdW, Coul, NumRotors, RMSD, Score), (+23. 905, -26. 781, +1. 900, -28. 681, 14, 4. 230, -5. 987) Kcalmol. (Figure S3b), (Figure S3e) On the other hand, the RoccuffirnaTM quantum thinking novel scaffold reproduced a negative docking effect while interacting with negative docking energies onto the binding sites of the protein targets of (PDB:6xs6) with the docking energy values of (T. Energy, I. Energy, vdW, Coul, NumRotors, RMSD, Score), (-84. 576, -0. 705, $-7,064,-0.705,12,8.613,16.203) \mathrm{Kcalmol}$. The Roccuffirna small molecule bonding interactions between its active pharmacophoric residues of 2S, 5R, 6R) -6- ((2S) -2-amino-2-phenylacetamido) -3, 3dimethyl-7- oxo-4-thia-1- azabicyclo (3. 2. 0) heptane-2-carbonyloxy), (\{ ((2-amino-6-oxo-6, 9-dihydro-3Hpurin-9-yl) oxy), (hydroxy) phosphoryl\}oxy) phosphinicacid-oxy) butyl) -6'-oxo-1', 4', 5', 6'-tetrahydro2lambda5-spiro (oxaziridine-2, 9'-purin) -2-ylium. (Fig \Ss2a, 2b, 2c, 2d, 2e, 2f, 3a, 3b, 3c), (R) -\{((2R) -1((3S, 4'R, 5'S) -2'-amino-6'-oxo- 1', 4', 5', 6'-tetrahydro-2lambda5-spiro (oxaziridin2, 9'- purin) -3-yl) butan2-yl) oxy\} (\{ ((2R, 4R) -2- ((1-fluoroethenyl), (hydroxymethyl) ami no) -5-oxa-1 lambda3-thia-3azabicyclo (2. 1. 0) pentan-3-yl) methoxy\}) hydroxy (pyrrolidin-1-yl) phosphanium are observed to be engaged in the construction of Hydrophobic interactions, and Water bridges with (PDB:6lu7) protein targets within the 02J:C:1 (02J) Interacting chains A of the amino acid sequence of V-S-VAL-116, V-M- 
THR-118, V-S-THR-118, V-M-PRO-84, V-S-PRO-84, V-M-ASN-85, V-M-ASN-114, V-M-THR-115, V-S-THR-115, with the docking energy values of $(-3.53,-2369,-1303,-10.425,-3.420,-72.447,-13.394,-3.190,-70$. $551)$ Kcalmol. (Figure S3b) Binding interactions between the entire ((2S, 5R, 6R) -6- ((2S) -2-amino-2phenylacetamido) -3, 3-dimethyl-7- oxo-4-thia-1-azabic yclo (3. 2. 0) heptane-2-carbonyloxy), (\{ ((2amino-6-oxo-6, 9-dihydro-3H-purin-9-y I) oxy), (hydroxy) phosphoryl\} oxy) phosphinic acid ((\{ ((2R, 3S, 5R) - $\{((1 S, 4 S)$-5-oxabicyclo (2. 1. 0) pentan-2-ylidene) $\{$ (cyano (\{2, 6-diazabicyclo (3. 1. 0) hex-1- en-6yl\}) phosphanyl), (fluoro) methyl\} -lambda6-sulfanyl\}one5- (2-amino-6- oxo-1, 6-dihydro -3lambda4, 7lambda4, 9lambda4- purin-9-yl) -3-hydroxyoxolan-2-yl) pharmacophoric residues of the Roccuffirna chemical design when docked onto the SARS-COV-2 protein sites are also shown to be interacted inside the active domains of the (PDB:6yb7) protein targets with in the binding cavities of the amino acid sequences of (V-S-LYS-5, V-S-TYR-126, V-S-ARG-131, V-S-LYS-137, V-S-GLU-288, V-S-GLU-290 with the docking energy values of (-6. 04578, -10. 1246, -4. 45654, -5. 54142, -8. 60669, - 11. 7695) Kcalmol respectively. The docking poses of the merged ((2S, 5R, 6R) -6- ((2S) -2-amino-2- phenylacetamido) -3, 3dimethyl-7-oxo- 4-thia-1-azabicyclo (3. 2. 0) heptane-2-carbonyloxy), (\{ ((2-amino-6-oxo-6, 9-dihydro-3Hpurin-9-y I) oxy), (hydroxy) phosphoryl\} oxy) phosphinic acid $\{((1 S, 4 S)$-5-oxabicyclo (2. 1. 0) pentan-2ylidene) $\{$ (cyano (\{2, 6-diazabicyclo (3. 1. 0) hex-1-en-6-yl\}) phosphanyl) (fluoro) methyl\} - lambda6sulfanyl\}one (hydroxy) phosphoryl) oxy) oxolan-2-yl) methoxy\} (hydroxy) phosphoryl) oxy) - 5- (2-amino6- oxo-1, 6-dihydro-3lambda4, 7lambda4, 9lambda4- purin -9-yl) oxolan-2-yl) methoxy\}phosphonic acid octatetracontahydrogen chemical residues of the entire Roccuffirna chemical structure when docked onto the SARS-COV-2 protein binding sites of the (PDB:3fqq) protein targets occupied the binding sites of the electrostatic surface of the active pocket cavities of the (PDB:3fqq) protein targets as bound to the Roccuffirna small molecule inside the binding domains of the amino acid sequences of the V-M-ARG-112, V-S-ARG-112, V-S-HIS-159, V-M-TYR-161, V-M-ARG-112, V-S-HIS-159 with the docking energy values of $(-87.2$ and $-5.9744,-11.9828,-13.3388,-4.9979,-5.08108,-13.4218)$ Kcalmol docking energy values respectively. Moreover, $\{((1 S, 4 S)$-5-oxabicyclo (2. 1. 0) pentan-2-ylidene) \{ (cyano (\{2, 6-diazabicyclo (3. 1. 0) hex-1-en-6-yl\}) phosphanyl) (fluoro) methyl\} - lambda6-sulfanyl\}one $\{((R)$ - (aminooxy), ((1'S, 1"S, 3S, 3'S, 4S, 5"S) -3'- (aminooxy) -5"-\{N- ((1R) -1, 2- ((2S, 5R, 6R) -6- ((2S) -2- amino-2-phenylacetamido) -3, 3- dimethyl-7-oxo-4-thia-1- azabicyclo (3. 2. 0) heptane-2- carbonyloxy), (\{ ((2-amino-6-oxo-6, 9-dihydro3H-purin-9-yl) oxy) (hydroxy) phosphoryl\} oxy) phosphinic acid) formamido\}-1'lambda5, 1"lambda5, 4"lambda6-dispiro (1, 2-oxazetidine- 3, 2'-phosphirane- 1', 3"- (6) oxa- (4lambda6) thia- (1lambda5) aza(3lambda5) phospha tetracyclo (3. 2. 0. 01, 4. 02, 4) heptane) -1' (4"), 1"-dien-4-

yl) phosphanyl) methylidene\}amino) - 3lambda4, 7lambda4, 9lambda4-purin-9-yl\} -3- ((\{ ((2R, 3S, 5R) -5(2-amino-6-oxo-1, 6-dihydro-\{ ((1S, 4S) -5-oxabicyclo (2. 1. 0) pentan-2-ylidene) $\{$ (cyan o $(\{2,6$ diazabicyclo (3. 1. 0) hex-1-en-6-yl\}) phosphanyl), (fluoro) methyl\}-lambda6-sulfanyl\}one3lambda 4, 7lambda4, 9lambda4-purin- 9-yl) -3-hydroxyoxolan- 2-yl) methoxy\}contact residues of the Roccuffirna chemical structure reproducedhydrophobic interactions against the 165 MET-A amino acid residue with the docking energy valuesof $(-3.90,-4673,-19.389,-17.775,-28.688,-16.611,-16.152,-26.489)$ Kcalmol while generatingwater bridges when docked onto the 401 X77A binding sites of the (PDB:3fqq) protein targets of the amino acid sequences of V-S-PRO-413, V-S-ASP-415, V-M-PRO-450, V-S-PRO-450, VS-PHE-451, V-S-GLU-502 with the negative docking energy values of (-76 and -5. 94924, -8. 74444, -6. 
$95118,-6.43726,-9.70804-6.23612)$ Kcalmol respectively. Additionally, the pharmacophoric contact residues of the Roccuffirna's $\{((1 S, 4 S)$-5-oxabicyclo (2. 1. 0) pentan-2-ylidene) $\{$ (cyano $(\{2,6$ diazabicyclo (3. 1. 0) hex-1-en-6-yl\}) phosphanyl), (fluoro) methyl\}-lambda6-sulfanyl\}one chemical structure when docked onto the SARS-COV-2 protein binding sites of the (PDB:6xs6) protein targets occupied the entire binding site of the electrostatic surfaces of the active site pocket of the (PDB:6xs6) domains inside the binding cavities of the amino acid sequences of the V-M-PHE-592, V-SPHE-592, V-M-GLY-614, V-M-VAL-615, V-M-ASN-616, V-S-ASN-616, V-S-GLN-644, V-M-ARG-646 with the docking energy values of -81.5 and $-5.45629,-4.42842,-11.2087,-9.94286,-6.5582,-7.35307,-5$. $32091,-5.63271$ docking energy values respectively. Gemdock docking analysis indicated that the contact residues of the Roccuffirna chemical structure hit the SARS-COV-2 protein binding sites when docked onto the (PDB:2g9t) protein targets. Moreover, the electrostatic surfaces of active pocket sites inside the (PDB:2g9t) protein targets when bound to the Roccuffirna small molecule ((2S, 5R, 6R) -6((2S) -2-amino- 2-phenylacetamido) -3, 3-dimethyl-7-oxo- 4-thia- 1- azabicyclo (3. 2. 0 ) heptane-2carbonyloxy), (\{ ((2-amino-6-oxo-6, 9-dihydro -3H-purin-9-yl) oxy), (hydroxy) phosphoryl\} oxy) phosphinic acid (hydroxy) phosphoryl) oxy) oxolan-2-yl) methoxy\} (hydroxy) phosphoryl) oxy) -5- (2-amino-6-oxo-1, 6-dihydro-3lambda4, 7lambda4, 9 lambda4-purin-9-yl) oxolan-2-yl) methoxy\}phosphonic acid octatetracontahydrogen residues inside the binding cavities of the amino acid sequences of the V-S-VAL116, V-M-THR-118, V-S-THR-118, V-M-PRO-84, V-S-PRO-84, V-M-ASN-85, V-M-ASN-114, V-M-THR-115, V-STHR-115, V-M-VAL-116 with the docking energy values of $(-86.6$ and $-4.78161,-5.02437,-5.37485,-6$. $01569,-4.08585,-9.1855,-4.79454,-5.5449,-4.52233,-4.53177)$ Kcalmol respectively. In addition, the Roccuffirna'Small molecule contact residues of the ((2S, 5R, 6R) -6- ((2S) -2-amino-2-phenylacetamido) 3, 3-dimethyl-7-o xo-4-thia-1-azabicyclo (3. 2. 0) hept ane-2-carbonyloxy) (\{ ((2-amino-6-oxo-6, 9-dihydro3H-purin-9-yl) oxy), (hydroxy) phosphoryl\}oxy) pho sphinic acid- (1S, 4S) -5-oxabicyclo*2. 1. 0+pentan2 ((2S, 5R, 6R) -6- ((2S) -2-amino-2-phenylacetamido) -3, 3-dimethyl-7-oxo-4-thia-1-azabicyclo (3. 2. $0)$ heptane-2-carbonyloxy), (\{ ((2-amino-6-oxo-6, 9-dihydro-3H-purin-9-yl) oxy) (hydroxy) phosphoryl\} oxy) phosphinic acid -ylidene+, *cyano (2, 6- diazabicyclo*3. 1. 0+hex-1- en-6yl-) phosphanyl+ (fluoro) methyl-lambda6-sulfanyl-one (( $((2 R, 3 S, 5 R)$-5- (2-amino-6-oxo- 1, 6-dihydro3lambda4, 7lambda4, 9lambda4- purin -9-yl) -3-hydroxyoxolan-2-yl) methoxy\} pharmacophoric components interacted with Hydrophobic Interactions with in the binding pockets of the PJE:C:5 (PJE-010 + 010:C:6 Interacting chains of the A, 25THR, A6, 010C binding sites with the negative docking energy values of $(-3.73,-2415,-179,-7.156,-21.406,-66.898,-8.709,-22.779,-70.002) \mathrm{K}$ almolA and while generating Hydrogen Bondsinside the binding cavities of the $26 \mathrm{THR}, \mathrm{A6}, 010 \mathrm{C}$ amino acid with the negative docking energy values of $(-3.81,-2415,-186,-7.156,-21.406,-66.898,-6.155,-24.392,-64$. 757) $\mathrm{Kcal} / \mathrm{mol}$. It also involved in the generation of Hydrogen Bondswith the peptide backbone of the amino acid of the $143 \mathrm{GLY}, \mathrm{A6}, 010 \mathrm{C} 03$ binding domains with the docking energies of $(-93,-2.80,-145$. $29,-1105,-2411,-8.911,-17.849,-65.703,-8.918,-17.918,-62.905)$ Kcalmol inside the PJE, C2 16, N3, 1266,02 binding pockets of the amino acid of the 164, HIS, A5, with pure negative docking energy values of $(-3.07,-153.73,2-408,-12.282,-14.994,-67.123,-15.161,-1-5.336,-68.144)$ Kcalmol (Figure S3c), (Figure S3f). (Figure S3d) Our Roccuffirna prototype generated ION ZN (zinc ion) ZN-A-901 metal complexes inside the A DOZ Interacting chains of the B 4756 protein side chains among the Zn 2674 
trigonal and pyramidal binding sites in the (PDB:3fqq) protein targets with in the non-structural protein of the amino acid of the 41 HIS B 902 with the docking energy values of $(-51.015,-53.952,-41.217,-52$. $956,-53.584,-41.918) \mathrm{Kcalmol}$ indicating that this drug novelty may serve as a potential hepatitis $\mathrm{C}$ virus inhibitor against the novel dimeric form of this HCV NS5A protein domain I. (Figure S4a), (Figure $\mathrm{S} 4 \mathrm{~b})$ The same prototype is escaping to another dimension as it was designed using micro-Black Hole geometry directions and is capable for the producing ZN-B-902 metal complex interactions when docked with in the BD-A-2 Interacting chains inside the B Interacting chains of the (PDB:3fqq) protein targets of the (non-structural protein 5a) targets. (Figure S4c) The Roccuffirna chemical structure also interacted with the GOL (Glycerol) GOL-A-1 Interacting chains of the A, B binding sites into the same regions of the (non-structural protein 5a) (PDB:3fqq) active sites while contacting the whole regions of the DMS (Dimethyl Sulfoxide), and DMS-A-803 binding sites in 6 lu7 (3c-likeproteinase) Interacting chains. The Roccuffirna's chemical entities of the C8- ( $(\{((2 R, 3 R, 4 R)-2-((1-$ fluoroethenyl) (hydroxymethyl) amino) -spiro (oxaziridine-2, 9'-purin) -3-yl) butan-2-yl) oxy\} (\{ ((2R, 4R) -2- ((1fluoroethenyl) is involved in the formation of hydrogen bondsinside the 401 X77 A 2216 Nam 02 binding cavities of the 143 GLY A amino acid with docking energy values of $(-2.17,-2.94,-148.03,-4682,-19$. $635,-22.244,-29.036,-18.779,24.455,-30.773)$ KcalmolA. More specifically, the Remdesivir small molecule generated docking energies of the $(0,0,0,2 .+41148,-5.69599,0,-8.7971,-0.00202603,0,0$, $-4.53782,29.6984,-3.38875,-5.17451,-6.22961,-3.3889,-9.25813,-0.35774,-3.91578,+15.1513,-2$. $5505,0,-0.321802) \mathrm{Kcalmol}$ when docked with in the binding pockets of the amino acidsof H-S-ARG-555 H-S-ASP-623 H-M-F86-101 V-S-ASP-452 V-S-LYS-551 V-M-ARG-553 V-S-ARG-553 V-M-ALA-554 V-M-ARG555 V-S-ARG-555 V-M-ASP-618 V-S-ASP-618 V-M-TYR-619 V-M-PRO-620 V-S-PRO-620 V-M-LYS-621 V-SLYS-621 V-M-ASP-623 V-S-ASP-623 V-S-ARG-624 V-S- MG-1004 V-M-F86-101 V-M-F86-101 residues of the (PDB:7bv2) protein targets. (Table s2), (Table s3), (Table s4) On the other hand in this drug design project we applied general solution of the wave equation, including the non-formalizable solutions as molecular modification strategies to generate the Roccuffirna QMMM drug design which is capable of interacting onto the binding domains of the cav7bv2_POP protein targets of the (PDB:7bv2) with the highest docking energy of the $-84.3 \mathrm{Kcalmol}$ while interacting with the docking energies of $(-4.32839,-7$. $23314,-16.1584,-2.31648,-3.36038,-0.703894,-2.01058,-17.7135,0,0,-0.014892,-0.074521,-4$. $10748,-0.807205,-8.45592,-1.50648,-7.08011,-3.05006) \mathrm{Kcalmol}$ when hits the interacting binding sites of the amino acid sequence of H-S-ARG-555 H-S-ASP-623 H-M-F86-101, V-S-ASP-452 V-S-LYS-551VM-ARG-553 V-S-ARG-553 V-M-ALA-554 V-M-ARG-555 V-S-ARG-555 V-M-ASP-618 V-S-ASP-618 V-M-TYR619 V-M-PRO-620 V-S-PRO-620 V-M-LYS-621 V-S-LYS-621 V-M-ASP-623 V-S-ASP-623 V-S-ARG-624 V-SMG-1004 V-M-F86-101 V-M-F86-101 of the protein targets of the (PDB:7bv2). Our in-house Roccuffirna chemical structure when docked onto the SARS-COV-2 protein binding sites of the (PDB:1xak) protein targets generated hydrophobic interactions with in the electrostatic surface of the active pockets of the amino acid sequences of the V-S-HIS-4, V-S-LEU-16, V-M-LYS-17, V-S-LYS-17, V-M-PRO-19, with the total docking energy of the (-64. 8 and -12. 0222, -4. 479, -8. 17046, -6. 49062, -4. 42519) Kcalmol proposing that our multi-targeting chemical design may reproduce an inhibitory effect for the down regulation of the expression levels of the SARS-CORONAVIRUS ORF7A accessory protein. (Figure S4e) The Roccuffirna's pharmacophoric elements of the $\{((2 R, 3 S, 5 R)-3-((\{((2 R, 3 S, 5 R)-5-\{2$-amino-6- $((E)-\{((R)-($ aminooxy), 
((1'S, 1"S, 3S, 3'S, 4S, 5"S) -3'- (aminooxy) -5"-\{N- ((1R) -1, 2- ((2S, 5R, 6R) -6- ((2S) -2-amino-2-

phenylacetamido) -3, 3-dimethyl-7-oxo-4-thia-1-azabicyclo (3. 2. 0) heptane-2-carbonyloxy), (\{ ((2-amino6-oxo-6, 9-dihydro-3H-purin-9-yl) oxy) (hydroxy) phosphoryl\} oxy) phosphinic acid) formamido\} -

1'lambda5, 1" lambda5, 4"lambda6-dispiro (1, 2-oxazetidine- 3, 2'- phosphirane-1', 3"- (6) oxa-

(4lambda6) \{ ((1S, 4S) -5-oxabicyclo (2. 1. 0) pentan-2-ylidene) \{ (cyano (\{2, 6-diazabicyclo (3. 1. 0) hex1-en-6-yl\}) phosphanyl), (fluoro) methyl\}- lambda6- sulfanyl\}onethia- (1lambda5) aza-

(3lambda5phosphatetracyclo (3. 2. 0. 01, 4. 02, 4) heptane) -1' (4"), 1"-dien-4-

yl) phosphanyl) methylidene\}amino) -3lambda4, 7lambda4, 9lambda4-purin-9-yl\}-3- ((\{ ((2R, 3S, 5R) -5-

(2-amino-6-oxo-1, 6-dihydro-3lambda4, 7lambda4, 9lambda4-purin-9-yl) -3-hydroxyoxolan-2-

yl) methoxy\} (hydro xy) phosphoryl) oxy) oxolan-2-yl) methoxy\} (hydroxy) phosphoryl) oxy) -5- (2-amino6-oxo-1, 6-dihydro-3lambda4, 7lambda4, 9lambda4-purin-9-yl) oxolan-2-yl) methoxy\} phosphonic acid octa tetraconta hydrogen contact residues docked onto the SARS-COV-2 protein binding sites of the (PDB:6lu7) protein targets inside the binding cavities of the amino acid sequences of the V-M-ASN-142, VS-ASN-142, V-M-MET-165, V-S-MET-165, V-M-GLU-166, V-S-GLU-166, V-M-LEU-167, V-M-PRO-168, V-S-PRO168, V-M-ARG-188, V-M-GLN-189, V-S-GLN-189 with the docking energy values of (-96. 2, and -6. 29322, -5 . $82129,-7.21251,-4.2141,-5.18029,-12.1235,-4.71777,-6.9452,-4.94292,-5.31481,-5.14926,-11$. 2353) Kcalmol while reproducing an inhibitory docking energy effect against the crucial sequence of the amino acidsof the V-M-LEU-167, V-M-PRO-168, V-M-THR-190, V-M-ALA-191, V-M-ALA-2 with the docking energy values of $(-50.1$, and $-5.74191,-5.1612,-5.55901,-5.4395,-6.88909)$ Kcalmol indicating that our Quantum Kerr- (A) Ds Galilean Myers-Perr designed small molecule could serve as a down regulator of the expression levels of the crystal structure of COVID-19 main protease. (Figure S5c) The RoccuffirnaTM\{ ((1S, 4S) -5-oxabicyclo (2. 1. 0) pentan-2-ylidene) \{ (cyano (\{2, 6-diazabicyclo (3. 1. 0) hex-1 -en-6-yl\}) phosphanyl), (fluoro) methyl\} - lambda6-sulfanyl\}one methox (hydroxy) phosphoryl) oxy) oxolan-2-yl) methoxy\} (hydroxy) phosphoryl) oxy) -active residues bindsthe whole binding cavities of the (PDB:6LU7) protein targets inside the 02J-C-1 02J (5Methylisoxazole-3-carboxylic acid) 02J-C-1 Interacting chains (Figure S5d) when constructing PJE-C-5 PJE ligand composites and PJE-C-5 PJE:C:5, 010:C:6 pharmacophoric residues, water bridges and hydrogen bonds (Figure S5e), (Figure S5f), (Figure S5g) onto the PJE-C-5 PJE ligand composites PJE-C5PJE:C:5, 010:C:6 domains. (Figure S6a). Roccuffirna'sdrug design interacts with the ZN-B-902 Interacting chains of the $3 \mathrm{fqq}$ (non-structural protein $5 \mathrm{a}$ ) triangular side chains (Figure S6b) while reproducing hydrogen bondsinside the BD-A-2 Interacting chains of the (PDB:3fqq) (non-structural protein 5a) protein sides (Figure S6c) as bound with the GOL (Glycerol) GOL-A-1:A, B Interacting chains of the (PDB:3fqq) (non-structural protein 5a) protein targets accordingly. (Figure S6d). Chemical bridges of the active pharmacophores of the $\{((1 S, 4 S)$-5-oxabicyclo (2. 1. 0) pentan-2-ylidene) \{ (cyano ( $\{2,6$-diazabicyclo (3. 1. 0) hex-1- en-6-yl\}) phosphanyl), (fluoro) methyl\}-lambda6-sulfanyl\}one ((1'S, 1"S, 3S, 3'S, 4S, 5"S) -3'(aminooxy) -5"-\{N- ((1R) -1, 2- ((2S, 5R, 6R) - 6- ((2S) -2-amino-2- phenylacetamido) - 3, 3-dimethyl-7-oxo4-thia-1- azabicyclo (3. 2. 0) heptane-2-carbonyloxy), (\{ ((2-amino-6- oxo-6, 9-dihydro-3H-purin-9-yl) oxy) (hydroxy) phosphoryl\} oxy) phosphinic acid) formamido\}-1'lambda5, 1 "lambda 5, 4"lambda6dispiro residues ofthe Remdesivir drug when docked onto the SARS-COV-2 protein binding sites of the (PDB:1xak) protein targets inside the active site pocket of the (PDB:1xak) protein target generated 
less dockingenergies of 7, 45-fold times (Figure S6e) when compared to the Roccuffirna small molecule. (Figure S6f) Roccuffirna geometrical descriptor calculations and (Figure S6a) a CoMFA contour map analysis of the electrostatic regions were also performed around Roccuffirna chemical structure showing that the $((2 R, 3 S, 5 R)-3-((\{((2 R, 3 S, 5 R)-5$-\{2-amino- 6- $((E)-\{((R)-(a m i n o o x y))(1,2-$ oxazetidine- 3, 2'- phosphirane- 1', 3"- (6) oxa- (4lambda6) thia- (1lambda5) aza(3lambda5) phosphatetracyclo (3. 2. 0. 01, 4. 02, 4) heptane) -1 ' (4"), 1"-die n-4yl) phosphanyl) methylidene\}amino) -3lambda4, 7lambda 4, 9lambda4-purin- 9-yl\} -3- ((\{ ((2R, 3S, 5R) -5(2-amino- 6-oxo-1, 6-dihydro-3la mbda4, 7lambda4, 9lambda4-purin-9-yl) -3-h ydro xyoxolan-2 -yl) methoxy\} (hydroxy) phosphoryl) oxy) oxolan-2-yl) methoxy\} (hydroxy) phosphoryl) o xy) -5- (2amino-6-oxo-1, 6-dihydro-3 lambda4, 7lambda4, 9 lambda4-purin-9-yl) oxolan-2-yl) methoxy\}p hosphonic acid octatetraconta hydrogen contact residues of the Roccuffirna small molecule when docked onto theSARS-COV-2 protein targets, (PDB:6lu7) (Figure S6b) while co-interacting onto the SARSCOV-2protein binding sites of the (PDB:1 xak) protein targets simultaneously with in the amino acid sequences of the V-S-HIS-4, V-S-LEU-16, V-M-LYS-17, V-S-LYS-17, V-M-PRO-19, with the totaldocking energy of the (-64. 8 and $-12.0222,-4.479,-8.17046,-6.49062,-4.42519)$ respectively. (Figure S6b) More docking experiments were performed between the scaffold residues of theRoccuffirna chemical structure onto the SARS-COV-2 protein binding sites of the (PDB:6lu7) protein targets inside the active pocket cavities of the (PDB:6lu7) protein targets as bound to theRoccuffirna small molecule $\{((2 R, 3 S, 5 R)-3-$ $((\{((2 R, 3 S, 5 R)-5-\{2$-amino-6- ((E) - yl) methoxy\} (hydroxy) phosphoryl) oxy) -5- (2- $\{((1 S, 4 S)$-5oxabicyclo (2. 1. 0) pentan-2-ylidene) $\{$ (cyano (\{2, 6-diazabicyclo (3. 1. 0) hex-1-en- 6-yl $\}$ ) phosphanyl) (fluoro) methyl\} -lambda6- sulfanyl\}one amino-6-oxo-1, 6-dihydro-3lambda4, 7 lambda4, 9 lambda4purin-9 -yl) oxolan-2-yl) methoxy\}phosphonic acid octa tetraconta hydrogen chemical bridges inside the binding cavities of the amino acid sequences of the V-M-LEU-167, V-M-PRO-168, V-M-THR-190, V-M-ALA191, V-M-ALA-2 with the docking energy values of $(-50.1$, and $-5.74191,-5.1612,-5.55901,-5.4395,-6$. 88909) Kcalmol respectively (Figure S6c) while co-generating a parallel docking energy effect onto the SARS-COV-2 protein binding sites of the (PDB:5r80) protein targets inside the binding cavities of the amino acid sequences of the V-M-ASN-142, V-S-ASN-142, V-M-MET-165, V-S-MET-165, V-M-GLU-166, V-SGLU-166, V-M-LEU-167, V-M-PRO-168, V-S-PRO-168, V-M-ARG-188, V-M-GLN-189, V-S-GLN-189 with the docking energy values of $(-96.2$, and $-6.29322,-5.82129,-7.21251,-4.2141,-5.18029,-12.1235,-4$. $71777,-6.9452,-4.94292,-5.31481,-5.14926,-11.2353$ ) accordingly. (Figure S6d) The Roccuffirna drug design hits the SARS-COV-2 protein binding sites of the (PDB:6yb7) protein targets inside the binding cavities of the amino acid sequences ofV-S-LYS-5, V-S-TYR-126, V-S-ARG-131, V-S-LYS-137, V-S-GLU-288, V-S-GLU-290 with the docking energy values of (-71. 2 and -6. 04578, -10. 1246, - 4. 45654, -5. 54142, -8. $60669,-11.7695)$ Kcalmol respectively. (Figure S6e). In this paper, we used Galilean Transformation in Quantum Mechanics (22) while inS':L jj d 02J:C:1 (02J • $\otimes \cdots \ldots \ldots . . . . P J E-C-5 P J E: C: 5,010: C: 6 i$ ILL`d 02J:C:1 (02J • $\otimes \cdots \ldots . . . . . P J E-C-5 P J E: C: 5,010: C: 6 t y s^{\prime}====e$ 2h <p 02J:C:1 for the computer-aided design of the contact residues of the Roccuffirna chemical structure which is capable of interacting onto the SARS-COV-2 protein binding sites of the (PDB:3fqq) protein targets inside the Electrostatic surfaces of active site pocket of the (PDB:3fqq) protein targets as bound to the Roccuffirna small molecule inside the binding cavities of the amino acid sequences of the V-S-PRO-413, V-S-ASP-415, V-M-PRO-450, V-S-PRO- 
450, V-S-PHE-451, V-S-GLU-502 with the docking energy values of (-76 and -5. 94924, -8. 74444, -6. 95118, -6. 43726, -9. $70804-6.23612)$ Kcalmol docking energy values respectively. (Figure S5g) ((R) -

(aminooxy) ((1'S, 1"S, 3S, 3'S, 4S, 5"S) -3'- (aminooxy) -5"-\{N- ((1R) -1, 2- ((2S, 5R, 6R) -6- ((2S) -2-amino2- phenylacetamido) -3, 3- dimethyl-7-oxo- 4-thia-1- azabicyclo (3. 2. 0) heptane-2-carbonyloxy), (\{ ((2amino-6-oxo-6, 9-dihydro-3H-purin-9-yl) oxy) (hydroxy) phosphoryl\} oxy) phosphinic acid) formamido\}1'lambda5, 1"lambda5, 4" lambda6-dispiro (1, 2- oxazetidine-3, 2'-phosphirane -1', 3"- (6) oxa- ((2S, 5R, 6R) -6- ((2S) -2-amino-2- phenylacetamido) -3, 3-dimethyl-7-oxo-4-thia-1-azabicyclo (3. 2. 0) heptane-2car bonyloxy), (\{ ((2-amino-6-oxo-6, 9-dihydro-3H-purin-9-yl) oxy), (hydr oxy) phosphoryl\}oxy) phosphinicacid $\{((1 S, 4 S)$-5-oxabic yclo (2. 1. 0) pentan-2-ylidene) \{ (cyano (\{2, 6diazabicyclo (3. 1. 0) hex-1-en-6-y|\}) phosphanyl), (fluoro) methyl\} -lambda6sulfanyl\}one (4lambda6) thia- (1lambda5) aza- (3lambda5) contact residues (Figure S6f) of the Roccuffirna chemical structure were the generated when docked onto the SARS-COV-2 protein binding sites of the (PDB:6xs6) protein targets inside the active pocket sites of the (PDB:6xs6) protein targets bound to the Roccuffirna'Small molecule regions of the $\{((1 S, 4 S)-5$ - oxabicyclo (2. 1. 0) pentan- 2ylidene) $\{$ (cyano ( $\{2,6$-diazabicyclo (3. 1. 0) hex-1-en-6-yl\}) phosphanyl) (fluoro) methyl\} -lambda6sulfanyl\}oneinside the binding cavities of the amino acid sequences of the V-M-PHE-592, V-S-PHE-592, VM-GLY-614, V-M-VAL-615, V-M-ASN-616, V-S-ASN-616, V-S-GLN-644, V-M-ARG-646 with the docking energy values of $(-81.5$ and $-5.45629,-4.42842,-11.2087,-9.94286,-6.5582,-7.35307,-5.32091,-5$.

63271) KcalmolA (Figure S6f). Finally, docking energy comparative analysis has indicated to us that our innovative Roccuffirna small molecule generated a co-inhibitory binding energy effect when combined with the drugs of the baricitinib, valsartan, gemigliptin, raltegravir, doxycucline, colchicines, azathioprine, hydroxychloroquine, umifenovir, linoleic acid, ribavirin, eflornithine, cobicistat and the remdesivir when docked onto the same SARS-COV-2 protein targets.

\section{Discussion}

In this work we introduced an operationalfor malismand a relational reference frame theory that predicts a fundamental decoherence mechanism, by combining black-hole cryptography solutions to Einstein's Eqsfor perfor ming quantum communication, neural matrix factorizations, and putative ligand-receptor visualizations to apply quantum mechanics from the point of view of a reference frame translated to a quantum pharmacophoric system of ((2S, 5R, 6R) -6- ((2S) -2-amino-2- phenylacetamido) -3, 3- dimethyl7-oxo- 4-thia-1- azabicyclo (3. 2. 0) heptane-2-carbonyloxy), (\{ ((2-amino-6- oxo- 6, 9-dihydro-3H-purin-9yl) oxy) (hydroxy) phosphoryl\} oxy) phosphinic acid—chemical bridge, which we call quantum reference frame. $(31-42,43)$ This reference frame has an arrow of time emerges from a time-symmetric theory on virtual compound libraries and its own degrees of freedom, which can be in quantum superposition or entangled and evolve in time according to their own Hamiltonian with respect to the docking frame of reference. Moreover, our model adopts a relational view, according to which any reference frame is described as a quantum degree of freedom relatively to another reference frame, collapse of the wave packet and circumvents the problem of generalizing chemical characters to a quantum pharmacophoric system as the probability interpretation which was only ever applied to diagonal density operators. Hence, 
theframe of reference in this research paper by combining black-hole cryptography solutions to Einstein's Eqsfor perfor ming quantum cryptographic communications is a quantum system relative to the quantum reference frame of a pharmacophoric system of ((2S, 5R, 6R) - 6- ((2S) -2-amino-2phenylacetamido) -3, 3- dimethyl-7-oxo- 4-thia-1- azabicyclo (3. 2. 0) heptane-2- carbonyloxy), (\{ ((2amino-6-oxo- 6, 9-dihydro-3H-purin-9-yl) oxy) (hydroxy) phosphoryl\} oxy) phosphinic acid, much like the pharmacophoric system of ((2S, 5R, 6R) -6- ((2S) -2-amino-2- phenylacetamido) -3, 3- dimethyl-7-oxo4-thia-1- azabicyclo (3. 2. 0) heptane- 2-carbonyloxy), ( ( (2-amino-6-oxo- 6, 9-dihydro-3H-purin-9-yl) oxy) (hydroxy) phosphoryl\} oxy) phosphinic acid that interprets a quantum system relative to the laboratory frame. This allows us to avoid assuming the existence of an external perspective of an absolute reference frame and choose a generalized parity-swap operator which acts as a piece of information that enables the sender of a message to encrypt the message and the receiver of a message to decrypt the message (Hoffstein, Pipher, \& Silverman, 2008) in such a way that the solutions of the Eqs of motion of pharmacophoric system $C$ from the point of view of $A$ are of opposite sign to those of the Eqs of motion of pharmacophoric system of ((2S, 5R, 6R) - 6- ((2S) -2-amino-2- phenylacetamido) - 3, 3dimethyl-7-oxo- 4-thia-1- azabicyclo (3. 2. 0) heptane-2- carbonyloxy), (\{ ((2-amino-6- oxo-6, 9-dihydro3H-puri n-9-yl) oxy) (hydroxy) phosphoryl\} oxy) phosphinic acid from the point of view of C. (METHODS AND MATERIALS) (Scheme of Eqs. 1-210), (METHODS AND MATERIALS) (Cluster of Eqs. 1-104) (Table S10), (Table S11) (Figure S7), (Figure S8), (Table S14), (Figure S9) Moreover, additional docking energy validation studies indicated that our prototypes RoccuffirnaTM, RoccuttirnaTM, EplerotiffirnaTM innovative chemical structures, generated the highest rates of negative docking energy values when compared with the Amprenavir, Asunaprevir, Atazanavir, Boceprevir, Cytarabine, Darunavir, Ritonavir, Sorivudine, Taribavirin, Tenofovir, Valganciclovir, Vidarabine, Lopinavir, Sofosbuvir, Zanamivir, Penciclovir, Nelfinavir, Merimepodib, Maribavir, Indinavir, Inarigivir, Galidesivir, Famciclovir, Faldaprevir FDA approved antiviral drugs inside the binding cavities of the amino acid sequence of V-S-TYR-35, V-M-GLY-38, V-MALA-39, V-M-ASP-40, V-S-ASP-40, V-M-GLY-81, V-M-ARG-82, V-S-ARG-82, V-M-TYR-83, V-S-TYR-83, V-MMET-84, V-S-MET-84, V-M-SER-85, V-S-SER-85, V-M-ALA-86, V-S-LEU-87, V-M-THR-158, V-S-THR-158, V-MVAL-159, V-M-VAL-159 onto the SARS-COV-2 protein binding sites of the (PDB:6lu7) crystal structures of the COVID-19 main protease protein targets (Figure S10). More specifically, our drug design novelty named EplerotiffirnaTM when combined with RoccuffirnaTM and RoccuttirnaTM small molecules onto the active sites of the (PDB:6lu7) protein targets reproduced TotalEnergy, and VDW energy values of $(-497.176,-497.176)$, and free energy values of $(-4.07251,-0.908714,-0.566584,-2.00645,-6$. $56941,-1.11384,-4.37848,-2.707,-0.562$ 642, -0. 542996, -0. 115371, -8. 59621, -7. 2098, -5. 76026, -1. $09013,-4.80574,-7.37652,-6.91952,-5.32324,-3.75$ 622) Kcalmol respectively. More specifically, our drug design novelties named RoccuffirnaTM, RoccuttirnaTM, and EplerotiffirnaTM generated higher rates of negative docking energy scoring values when compared with Amprenavir, Asunaprevir, Atazanavir, Boceprevir, Cytarabine, Darunavir, Ritonavir, Sorivudine, Taribavirin, Tenofovir, Valganciclovir, Vidarabine, Lopinavir, Sofosbuvir, Zanamivir, Penciclovir, Nelfinavir, Merimepodib, Maribavir, Indinavir, Inarigivir, Galidesivir, Famciclovir, Faldaprevir FDA approved antiviral drugs against the PDB: 2amd (Figure S11a), PDB: 2gtb (Figure S11b), PDB: 2alv (Figure S11c), PDB: 2a5i (Figure S11d), PDB: 6wzu (Figure S11e), PDB: 5 r80 (Figure S11f), PDB: 5 ry7 (Figure S11g), PDB: 1 wof (Figure S11h), PDB: 1 uk4 (Figure 
S11i) SARS-COV-2 protease associated protein targets. Quantum cryptography not just scratched the surface of the quantum realm, as time goes on and knowledge progresses but seems to excel in many areaswhere the other methodsfail. However, the distance over which quantum cryptography can take place is still lacking.

\section{Concluding Remark}

In this original research paper, I write the Schrödinger equation in quantum reference frames. As a part of this project that the quantum canonical transformations are defined outside of the Hilbert space, we finally designed small molecules the RoccuffirnaTM's chemical structures that comprising the highest binding free energy docking values based on reliable comparisons of numerical cryptographical models with observed docking data by using Galilean Black Hole Transformations, Quantum chemistry phenomena, and Pharmacophoric Merging Hyper-algorithms. $(31,32,37-43)$ As a by-product of the fact that the quantum canonical transformations are defined outside of the Hilbert space, I was able of the QMMM construction of the Roccuffirna small molecule by introducing numerical cosmological calculations to investigate different quantum chemistry phenomena. Limitations still existed in the current study since Quantum computers are exceedingly difficult to engineer, build and program. QC could make current CADD tools more effective by helping to predict molecular properties with high accuracy. That can affect the development process in several ways, such as modeling how SARS-COV-2 proteins fold and how anti-viral drug candidates interact with biologically relevant proteins. I still believe that quantum computing is likely to transform only the very early stages of pharmaceutical R\&D over the coming decades but that it will provide near-term benefits as the technology matures. Considering that quantum cryptographical techniques will continue to advance drug discovery approaches to obtain the relational degrees of freedom from the very start physical degrees of freedom to be relational from the point of view of a chosen QRF. These Schrödinger inspired docking algorithms when combining with black-hole solution to Einstein's Eqs as seen from a QRF and other chemistry-theoretic tasks of the reference-frame transformation for finding topological descriptors, eigenvectors, and eigenvalues with advanced machine learning algorithms, such as the data mining to merge pharmacophoric elements used could be appropriate to design multi-targeted ligands for other diseases as well.

\section{Declarations}

\section{AVAILABILITY OF DATA AND MATERIALS}

The paper reports original code. Any additional information required to reanalyze the data reported in this paper is available from the lead contract upon request.

\section{COMPETING INTEREST}

No potential competing interest was reported by the author.

\section{FUNDING}


No funding received for this research article

\section{AUTHOR CONTRIBUTIONS}

Grigoriadis loannis's diverse contributions to the published work are accurate and agreed. Grigoriadis loannis has contributed to the below multiple roles:

- Conceptualization Ideas, formulation or evolution of overarching research goals and aims.

- Methodology, Development, or design of methodology; creation of models.

- Writing - Review \& Editing, Preparation, creation and presentation of the published work by those from the original research group, specifically critical review, commentary, or revision including pre-or post-publication stages.

- Visualization, Preparation, creation, and presentation of the published work, specifically visualization/data presentation.

- Supervision, Oversight and leadership responsibility for the research activity planning and execution, including mentorship external to the core team.

- Project administration, Management, and coordination responsibility for the research activity planning, and execution.

\section{ACKNOWLEDGMENTS}

I would like to express my special thanks of gratitude to my teacher (George I Grigoriadis Pharmacist) who gave me the golden opportunity to do this wonderful project on the Quantum Chemistry topic, which also helped me in doing a lot of Research and I came to know about so many new things I am thankful to them.

\section{AUTHOR'S INFORMATION}

Further information and requests for resources should be directed to and will be fulfilled by the lead contract, Grigoriadis loannis (jgrigoriadis@biogenea.gr).

\section{References}

1. Hui DS. Epidemic and Emerging Coronaviruses (Severe Acute Respiratory Syndrome and Middle East Respiratory Syndrome). ClinChestMed. 2017;38 (1) :71-86.

2. Zhu N, Zhang D, Wang W, Li X, Yang B, Song J, Novel Coronavirus from Patients with Pneumonia in China, 2019. N Engl J Med. 2020;382 (8) :727-33.

3. Paraskevis D, Kostaki EG, Magiorkinis G, Panayiotakopoulos G, Sourvinos G, TsiodraS. Full-genome evolutionary analysis of the novel coronavirus (2019-nCoV) rejects the hypothesis of emergence as a result of a recent recombination event. InfectGenetEvol. 2020;79:104212. 
4. Lu R, Zhao X, Li J, Niu P, Yang B, Wu H, G enomiccharacterisation and epidemiology of 2019 novel coronavirus: implications for virus origins and receptor binding. Lancet. 2020;395 (10224):565-74.

5. Mueller S. On DNA Signatures, Their Dual-Use Potential for GMO Counterfeiting, and a Cyber-Based Security Solution. FrontBioengBiotechnol. 2019;7:189. Published 2019 Aug 7. doi:10. 3389fbioe. 2019. 00189

6. Ziebuhr J. Molecular biology of severe acute respiratory syndrome coronavirus. CurrOpinMicrobiol. 2004;7 (4) :412-9.

7. WeisSR, Leibowitz JL. Coronavirus pathogenesis. Adv Virus Res. 2011;81:85-164.

8. Brian DA, Baric RS. Coronavirus genome structure and replication. Curr Top Microbiol Immunol. 2005;287:1-30.

9. Narayanan K, Huang C, Makino S. SARS coronavirus accessory proteins. Virus Res. 2008;133 (1) :113-21.

10. Arndt AL, Larson BJ, Hogue BG. A conserved domain in the coronavirus membrane protein tail is important for virus assembly. J Virol. 2010;84 (21) :11418-28.

11. Neuman BW, Kiss G, Kunding AH, Bhella D, Baksh MF, Connelly S, et al. A structural analysis of M protein in coronavirus assembly and morphology. J StructBiol. 2011;174 (1):11-22

12. Siu KL, Chan CP, Kok KH, Chiu-Yat Woo P, Jin DY. Suppression of innate antiviral response by severe acute respiratory syndrome coronavirus $\mathrm{M}$ protein is mediated through the first transmembrane domain. Cell Mol Immunol. 2014;11 (2) :141-9.

13. Schoeman D, Fielding BC. Coronavirus envelope protein: current knowledge. Virol J. 2019;16 (1):69.

14. Pilon, A. C., Valli, M., Dametto, A. C. et al. NuBBEDB: an updated database to uncover chemical and biological informationfrom Brazilian biodiversity. Sci Rep 7, 7215 (2017). https: // doi. org10. 1038s41598-017-07451-x

15. Ruch TR, Machamer CE. The coronavirus E protein: assembly andbeyond. Viruses. 2012;4 (3):36382.

16. McBride R, van Zyl M, Fielding BC. The coronavirus nucleocapsid is a multifunctional protein. Viruses. 2014;6 (8) :2991-3018.

17. Chang CK, Hou MH, Chang CF, Hsiao CD, Huang TH. The SARScoronavirus nucleocapsid protein--for ms and functions. Antiviral Res. 2014;103:39-50.

18. Yan R, Zhang Y, Li Y, Xia L, Guo Y, Zhou Q. Structural basis for therecognition of the SARS-CoV-2 by full-length human ACE2. Science. 2020.

19. Walls AC, Park YJ, Tortorici MA, Wall A, McGuire AT, Veesler D. Structure, Function, and Antigenicity of the SARS-CoV-2 Spike Glycoprotein. Cell. 2020.

20. Hoffmann M, Kleine-Weber H, Schroeder S, Kruger N, Herrler T, Erichsen S, et al. SARS-CoV-2 Cell Entry Dependson ACE2 and TMPRSS2 and Is Blocked by a Clinically Proven Protease Inhibitor. Cell. 2020. 
21. Kang H, Bhardwaj K, Li Y, Palaninathan S, Sacchettini J, Guarino L, et al. Biochemical and genetic analyses of murine hepatitis virus Nsp15 endoribonuclease. J Virol. 2007;81 (24):13587-97.

22. Bhardwaj K, Sun J, Holzenburg A, Guarino LA, Kao CC. RNA recognition and cleavage by the SARS coronavirus endoribonuclease. J Mol Biol. 2006;361 (2) :243-56.

23. Zhang L, Li L, Yan L, Ming Z, Jia Z, Lou Z, et al. Structural andBiochemical Characterization of Endoribonuclease Nsp15 Encoded by Middle East Respiratory Syndrome Coronavirus. J Virol. 2018;92 (22).

24. Ton AT, Gentile F, Hsing M, Ban F, Cherkasov A. Rapid Identification of Potential Inhibitors of SARSCoV-2 Main Protease by Deep Docking of 1. 3 Billion Compounds. Mollnfor m. 2020.

25. Waterhouse A, Bertoni M, Bienert S, Studer G, Tauriello G, Gumienny R, et al. SWISS-MODEL: homology modelling of protein structures and complexes. Nucleic AcidsRes. 2018;46 (W1) :W296W303.

26. Wishart DS, Feunang YD, Guo AC, Lo EJ, Marcu A, Grant JR, et al. DrugBank 5. 0: a major update to the DrugBank database for 2018. Nucleic AcidsRes. 2018;46 (D1) :D1074-D82.

27. Tchesnokov EP, Feng JY, Porter DP, Gotte M. Mechanism of Inhibition of Ebola Virus RNA-Dependent RNA Polymerase by Remdesivir. Viruses. 2019;11 (4).

28. Al-Tawfiq JA, Al-Homoud AH, Memish ZA. Remdesivir as a possibletherapeutic option for the COVID19. Travel Med Infect Dis. 2020:101615.

29. Poulin D, Yard J (2007) Dynamics of a quantum reference frame. N J Phys 9 (5) : 156.

30. Skotiniotis M, Toloui B, Durham IT, Sanders BC (2013) Quantum Frameness for CPT Symmetry. PhysRevLett 111 (2) : 020504.

31. Giacomini F, Castro-Ruiz E, Brukner Č. Quantum mechanics and the covariance of physical laws in quantum reference frames. Nat Commun. 2019;10 (1) :494. Published 2019 Jan 30. doi:10. 1038s41467-018-08155-0

32. Poulin D (2006) Toy model for a relational formulation of quantum theory. Int J TheorPhys 45 (7) : $1189-1215$.

33. Girelli F, Poulin D (2008) Quantum reference frames and deformed symmetries. PhysRev D 77 (10) : 104012.

34. Miyadera T, Loveridge L, Busch P (2016) Approximating relational observables by absolute quantities: a quantum accuracy-size trade-off. J Phys. A 49 (18) : 185301.

35. Loveridge L, Busch P, Miyadera T (2017) Relativity of quantum states and observables. EPL (EurophysLett) 117 (4) : 40004.

36. Kunduri HK, Lucietti J. Classification of Near-Horizon Geometries of Extremal Black Holes. LivingRevRelativ. 2013;16 (1) :8. doi:10. 12942/rr-2013-8

37. Yoshida N. formation of the first generation of stars and blackholes in the Universe. Proc JpnAcad Ser B Phys Biol Sci. 2019;95 (1) :17-28. doi:10. 2183pjab. 95. 002 
38. Bertschinger E. Simulations of Structure formation in the Universe. Annu. Rev. Astron. Astrophys. 1998;36:599-654. doi: 10.1146annurev. astro. 36. 1. 599. (CrossRef) (Google Scholar)

39. Birrell ND, Davies PCW. Quantum Fieldsin Curved Space. Cambridge, U. K. : Cambridge University Press; 1982. (GoogleScholar)

40. Baumgarte TD, Shapiro SL. On the Numerical Integration of Einstein SFieldEqs. Phys. Rev. D. 1999;59:024007. doi: 10. 1103PhysRevD. 59. 024007. (CrossRef) (GoogleScholar)

41. Anninos P, Masso J, Seidel E, Suen W-M, Tobias M. Dynamics of Gravitational Waves in 3D: for mulations, Methods, and Tests. Phys. Rev. D. 1997;56:842-858. doi:10. 1103PhysRevD. 56. 842. (CrossRef) (GoogleScholar)

42. Anninos P, Centrella J, Matzner R. Nonlinear Wave Solutions to the Planar Vacuum Einstein Eqs. Phys. Rev. D. 1991;43:1825-1838. doi: 10. 1103PhysRevD. 43. 1825. (PubMed) (CrossRef) (GoogleScholar)

43. Chou YC. A radiating Kerr black hole and Hawking radiation. Heliyon. 2020;6 (1) :e03336. Published 2020 Jan 31. doi:10. 1016j. heliyon. 2020. e03336

44. Marcus Johansson1 and Valera VeryazovAutomatic procedure for generating symmetry adapted wavefunctions. Division of Theoretical Chemistry, Lund University, Naturvetarvägen 14, P. O. B. 124, 22100 Lund, Sweden.

45. Chambers C M. The Cauchy Horizon in Black Hole-de Sitter Spacetimes. In: Burko L M, Ori A, editors. Internal Structure of Black Holes and Spacetime Singularities. Bristol: Institute of Physics; 1998. [ Google Scholar]

46. Compère G. The KerrCFT correspondence and its extensions. Living Rev Relativ. 2017;20 (1) :1. doi: 10. 1007s41114-017-0003-2. Epub 2017 Feb 27. PMID: 28690421 ; PMCID: PMC5479153.

47. Nalewajski, R. F. Quantum information descriptors in position and momentum spaces. J Math Chem 53, 1549-1575 (2015). https: // doi. org10. 1007s10910-015-0505-7

\section{Supplementary Files}

This is a list of supplementary files associated with this preprint. Click to download.

- RoccuffirnajCHINSTOCD2eMETHODSANDMATERIALS.docx

- SupplementaryAppendixRoccuffirnajJOMCIRTh.doc

- floatimage1.png 